\title{
A Numerical Model for the Propagation of Ultra-High Energy Cosmic Rays through Extragalactic Magnetic Fields
}

\author{
Rubens Pereira Costa Junior \\ Center for Natural Sciences and Humanities (CCNH) \\ Federal University of $A B C$ (UFABC) \\ 09210-580 Santo André, São Paulo, Brazil \\ E-mail: rubens.costa@ufabc.edu.br
}

\section{Marcelo Augusto Leigui de Oliveira* \\ Center for Natural Sciences and Humanities (CCNH) \\ Federal University of $A B C$ (UFABC) \\ 09210-580 Santo André, São Paulo, Brazil \\ E-mail: leigui@ufabc.edu.br}

\begin{abstract}
We present a novel numerical model for the propagation of ultra-high energy cosmic rays in the extragalactic medium. We take into account the diffusion of ultrarelativistic charged particles under the influence of the magnetic fields, and their energy losses. The magnetic fields are considered to be coherent within spheric cells, and with smooth transitions between them. The propagator presents a very high accuracy on calculating the particle tracking, on the order of $\lesssim 10^{-33}$ of relative error in energy, and $10^{-5}$ in Larmor radius.
\end{abstract}

35th International Cosmic Ray Conference - ICRC2017

10-20 July, 2017

Bexco, Busan, Korea

\footnotetext{
${ }^{*}$ Speaker.
} 


\section{Introduction}

The study of the propagation and the interactions of the Ultra-High Energy Cosmic Rays (UHECRs) through galactic and extragalactic media is of great importance in Cosmic Ray (CR) physics. During their propagation the UHECRs deflect in magnetic fields and interact with lowenergy photons and matter such that their original energy spectra, primary chemical compositions, and arrival directions are expected to be modified. Hence, in order to comprehend the UHECRs energy spectrum, their composition, and their origin, among other features, it is mandatory to deal with the problem of determining the magnitude and the structure of the cosmic magnetic fields they cross.

There are indications that, at the highest energies, the CRs are of extragalactic origin. Above the so-called ankle $\left(\sim 4.8 \times 10^{18} \mathrm{eV}\right)$, the energy spectrum hardens from $J(E) \propto E^{-3.26}$ to $J(E) \propto$ $E^{-2.6}[1]$, which can be associated to a transition from the galactic to the extragalactic origin.

The strength and morphology of the ExtraGalactic Magnetic Fields (EGMFs) remain largely unknown [2,3], due to the intrinsic difficulty to observe them [4]. However, we know they can have both uniform and random components. The observational limit placed on the uniform large-scale component $\langle B\rangle_{\mathrm{IGM}}$ is $\lesssim 10^{-11} \mathrm{G}$ and on the amplitude of the random component $B_{\mathrm{r}}$ is $\lesssim 1.0 \mathrm{nG}$, for a coherence length of $l_{\text {coh }}=1 \mathrm{Mpc}[4,5]$. The former can be, in principle, neglected and the largescale random magnetic fields are generally assumed to have a cellular structure. The magnetic field is supposed to be uniform over a domain of fixed size $\left(l_{\mathrm{coh}}\right)$, randomly changing its direction from one domain to another but keeping approximately the same strength [6].

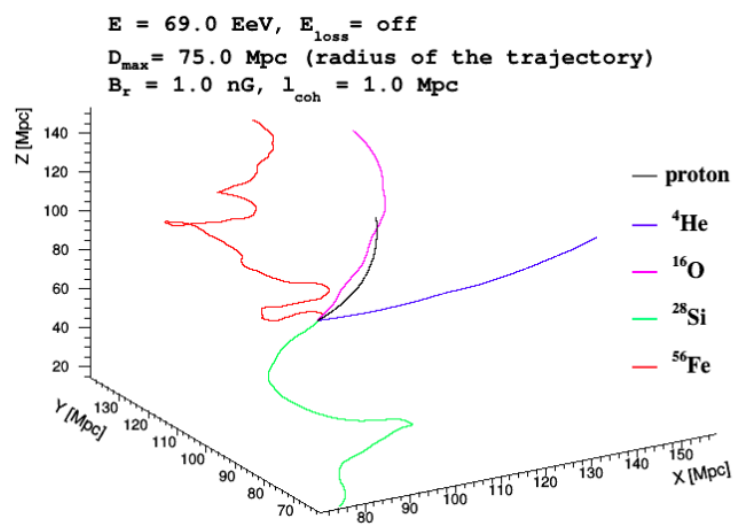

Figure 1: Trajectories of several nuclei with $E=69 \mathrm{EeV}$ in EGMFs by EGCRProp. We assumed $\vec{B}$ in cells of $l_{\mathrm{coh}}=1.0 \mathrm{Mpc}$ with random orientations, but constant intensities of $1.0 \mathrm{nG}$.

In this paper, we present a code, called EGCRProp, for tracking CRs through a medium of EGMFs of several Mpc. We assumed a cellular structure for the EGMFs, with random orientations, and smooth transitions between two neighboring cells. EGCRProp generates the coordinates of particles of different energies and compositions to be further displayed in a three-dimensional viewer (see figure 1). The EGCRProp package and its documentation are available for download from [7]. 


\section{The Code Structure}

EGCRProp is structured with two object-oriented codes written in $\mathrm{C}++$ : a tensorial magnetic field generator, and the propagator itself with the following classes:

- Mathematics: with methods for random number generation, and numerical interpolation;

- Geometry: with several vectorial methods like modulus, cross products, etc.;

- Dynamics: with dynamical methods like Lorentz force, particle's velocity, trajectory, or energy;

- Magnetic: with methods for calculationd of the magnetic fields details, and;

- Cosmology: with methods like Hubble distance, redshift, transverse comoving distance, etc.

The Universe is modelled as a cube of volume $(170 \mathrm{Mpc})^{3}$, within which unitary cells of diameter $l_{\mathrm{coh}}=1.0 \mathrm{Mpc}$, with constant magnetic fields of strength $B_{\mathrm{r}}=1.0 \mathrm{nG}$ and random orientations are given. In the beginning of an event $(t=0)$, a proton or a heavier nucleus of energy $E_{\mathrm{EeV}}$ is kicked from the Universe's center with the velocity's direction uniformly distributed in zenith and azimuth angle. Then, the particles are propagated and their deflections in the magnetic fields, as well as their energy losses due to the interactions with the background photons, are calculated.

\subsection{Extragalactic Magnetic Field Model}

As usually done in the majority of the studies of large-scale magnetic fields, we assumed the EGMFs to have a cellular structure, such that $\vec{B}$ is supposed to be almost constant over a coherence length $l_{\text {coh }}$, randomly changing its direction from one domain to another, but keeping the same strength. Thus, the tensorial magnetic field generator initially produces random orientations for the magnetic fields $\vec{B}(\theta, \phi)$ at the center of each coherence cell, placed in a cubic grid structure with lattice parameter of $l_{\mathrm{coh}}=1.0 \mathrm{Mpc}$.

Finally, we considered smooth transitions for the EGMFs between the cells (see figure 2). As the particle is travelling within the region distant $0.475 l_{\text {coh }}$ from the center of the nearest cell (dashed lines in figure 2) the magnetic field is the same as of the center of this cell. But outside this region, i.e. for $d>0.475 l_{\mathrm{coh}}$ from the center of the nearest cell, the orientation of the magnetic field $\vec{B}(\theta, \phi)$ is calculated by the weighted average:

$$
\begin{aligned}
& \theta=\left(\frac{\theta_{1}}{d_{1}}+\frac{\theta_{2}}{d_{2}}\right)\left(\frac{1}{d_{1}}+\frac{1}{d_{2}}\right)^{-1}, \\
& \phi=\left(\frac{\phi_{1}}{d_{1}}+\frac{\phi_{2}}{d_{2}}\right)\left(\frac{1}{d_{1}}+\frac{1}{d_{2}}\right)^{-1},
\end{aligned}
$$

where the weights are inversely proportional to the distances of the two nearest cells.

\subsection{The Particle Tracking Method}

In order to simulate the diffusion of charged particles in the magnetic fields, we integrate numerically the particle's equation of motion. If the electric field is absent (or negligible) for a 


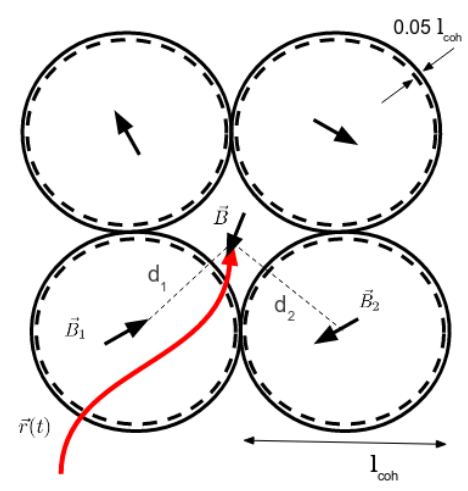

Figure 2: A sketch of a particle propagation in the transition region between the cells of constant magnetic fields, as modelled in our simulation.

charged particle of charge Ze traveling at the velocity $\vec{v}(t)$ at time $t$, subjected to a magnetic field $\vec{B}(\vec{r})$ along the trajectory $\vec{r}(t)$, the motion can be described by [8]

$$
\frac{d \vec{v}}{d t}=\vec{v} \times \vec{\omega}_{B}
$$

where $\vec{\omega}_{B}=\left(Z e / m_{0} \Gamma\right) \vec{B}$ is the cyclotron frequency for a particle of rest mass $m_{0}$ and (constant) Lorentz factor $\Gamma$. The solution for the velocity is given by

$$
\vec{v}(t)=v_{\|} \hat{\varepsilon}_{3}+\omega_{B} L_{R}\left(\hat{\varepsilon}_{1}-i \hat{\varepsilon}_{2}\right) e^{-i \omega_{B} t}
$$

which describes a helicoidal motion around the magnetic field line. The circular motion, perpendicular to the field line, is represented by the directions $\hat{\varepsilon}_{1}$ and $\hat{\varepsilon}_{2}$, where $v_{\perp}=\omega_{B} L_{R}$ is the perpendicular velocity component, and $L_{R}$ is the Larmor radius of the instantaneous circle. The uniform parallel translation is represented by the direction $\hat{\varepsilon}_{3}$ with velocity $v_{\|}$.

The Lorentz force $\vec{F}=m\left(\vec{v} \times \vec{\omega}_{B}\right)$ always acts perpendicularly to the direction of motion, making the particle to rotate around the magnetic field line.

The trajectory of the particle is then given by [8]

$$
\vec{r}(t)=r_{0}+v_{\|} t \hat{\varepsilon}_{3}+i L_{R}\left(\hat{\varepsilon}_{1}-i \hat{\varepsilon}_{2}\right) e^{-i \omega_{B} t} .
$$

At each time step, the particle's position and velocity are updated within a small fraction $\left(\xi \lesssim 10^{-3}\right)$ of the instantaneous period of revolution $T=2 \pi / \omega_{B}$.

\subsubsection{Machine Precision}

The software uses the features of the GNU MPFR library [9] to achieve the needed accuracy, mainly for the calculation of the particle's velocity parameter from the energy:

$$
\beta=\sqrt{1-\left(m_{0} c^{2} / E\right)^{2}},
$$

where $m_{0}$ and $E$ are, respectively, the particle's rest mass and total energy, and $c$ is the speed of light. 
The MPFR library is based on the GNU MP library [10] and allows using arbitrarily precise numbers instead of double precision numbers as standard. For energies above $\sim 10^{16.5} \mathrm{eV}$ the velocity parameter calculated with standard double precision is roughly $\beta=1$. The needed accuracy is achieved by using a $\mathrm{C}++$ interface for MPFR library [11], where all arithmetic and mathematical functions work via the MPFR library operating with mpfr-numbers. Consequently, a function or numerical code called by other classes works with arbitrary precision through the interface. EGCRProp runs with 50 digits of precision on its calculations.

\subsection{Energy Losses}

An effect of the expanding Universe is the constant change in the distance between two comoving objects. In the cosmology there are many ways to calculate the distance $D$ in an expanding Universe. In general, the Hubble constant is a function of the redshift $z, H(z)=H_{0} E(z)$, with the subscript "0" indicating the present epoch, and:

$$
E(z) \equiv \sqrt{\left.\left(1+z^{3}\right) \Omega_{M}+(1+z)^{2} \Omega_{k}+\Omega_{\Lambda}\right)}
$$

is a dimensionless function. For a flat space $\left(\Omega_{k}=0\right)$ the density parameter $\Omega$ follows the relation $\Omega=\Omega_{M}+\Omega_{\Lambda}=1$.

Also, the Hubble distance $D_{H}$ is defined as $D_{H} \equiv c / H_{0}$. In the expanding Universe, for a small redshift $z$, or a small velocity parameter $\beta$, it is possible to approximate:

$$
z \approx \beta=\frac{D}{D_{H}}
$$

If two objects are moving with the Hubble flow, the comoving distance:

$$
D_{C}=D_{H} \int_{0}^{z} \frac{d z^{\prime}}{E\left(z^{\prime}\right)}
$$

remains constant with the epoch.

To calculate the energy losses due to interactions with the background photons, we parametrised the mean energy loss per unit path length by the energy loss length $\chi_{\text {loss }}$, defined by:

$$
\chi_{\mathrm{loss}}(E)=\left|\frac{1}{E} \frac{d E}{d s}\right|^{-1}
$$

For large distances for the sources, cosmological effects must be taken into account. Due to the higher Cosmic Microwave Background (CMB) at a previous epoch, the photon density and the temperature at a distance corresponding to a redshift $z$ are, respectively, $n_{\gamma} \propto(1+z)^{3}$ and $T_{\mathrm{CMB}} \propto(1+z)^{3}$. As a consequence, the energy loss length at the redshift $z$ can be obtained from the value in the local Universe $(z=0)$ by using the scaling law [12]:

$$
\chi_{\mathrm{loss}}(E, z)=\frac{\chi_{\mathrm{loss}}[(1+z) E, z=0]}{(1+z)^{3}} .
$$

EGCRProp takes into account the energy loss length as shown in figure 3. The differences in the shape of the evolution of $\chi_{\text {loss }}$ between the proton and heavier nuclei cases are clearly visible. For complex nuclei, two sharp drops can be seen: the first one, at $\sim A \times 10^{18} \mathrm{eV}$, due to a combined effect of photodesintegration on far-infrared photons and pair production on CMB photons, and the second, at $\sim A \times 4 \times 10^{18} \mathrm{eV}$, is even sharper, due to photodisintegration on CMB photons. 


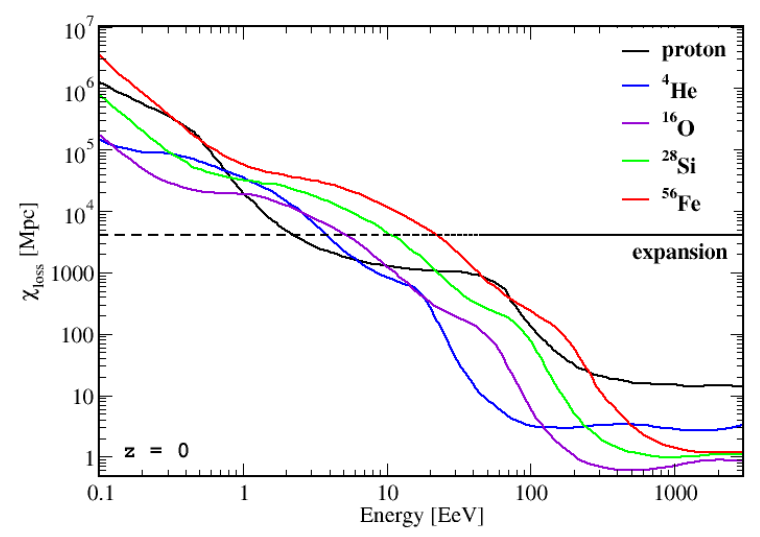

Figure 3: The energy evolution of the energy loss length, $\chi_{\text {loss }}$, for several nuclei at $z=0$ [13]. The contribution due to the Universe expansion is indicated in dotted line.

\section{Results}

To check the performance of our methods, we plotted in figure 4, the relative energy error as a function of the traveled distance through the EGMF for several types of nuclei with the same initial energy of $3.0 \mathrm{EeV}$, neglecting the energy losses. Each studied case has shown the same behavior

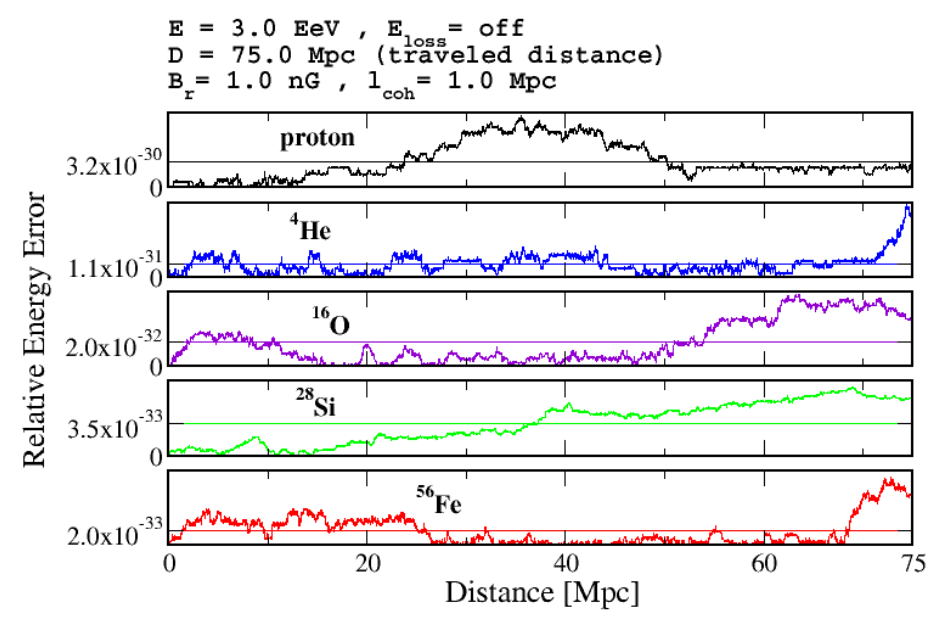

Figure 4: The relative error in energy as a function of distance for five different nuclei with initial energy of 3.0 EeV. The horizontal line indicates the average value.

on performing a circular trajectory with roughly $\sim 10^{-5} \%$ of relative error for the Larmor radius.

The distance amplifications from a rectilinear path, $\delta D / D_{0}$, are given in figures 5 , and 6 , respectively, as a function of final distance travelled (for three magnetic rigidities), and as a function of the magnetic rigidity (for some interesting final distances). The results are given for 1000 sim- 
ulations for each particle type, and the energy losses were also been turned off in these runs. As we can see, the distance increase for particles travelling up to $75 \mathrm{Mpc}$ through EGMFs reaches the level of $150 \%, 1.5 \%$, and $0.15 \%$, respectivelly, for initial energies of $3 \mathrm{EeV}, 30 \mathrm{EeV}$, and $100 \mathrm{EeV}$. These results clearly show when it is necessary to use a full three-dimensional simulation, like EGCRProp, for tracking UHECRs travelling across EGMFs.

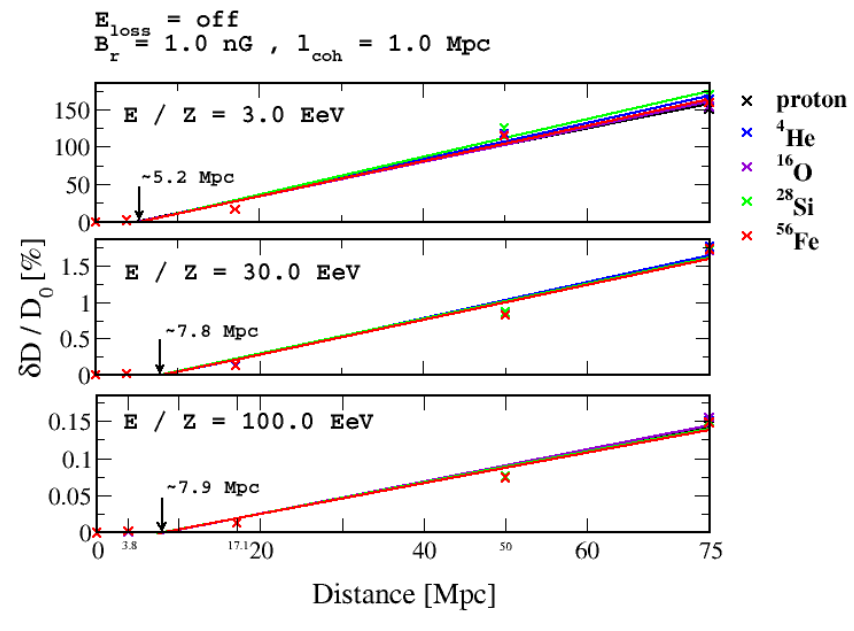

Figure 5: $\delta D / D_{0}$ as a function of final distance travelled, $D_{0}$, for 5 different nuclei, and 3 given magnetic rigidities, without energy losses. The arrows indicate the distances after which the magnetic deflexions are non-negligible.

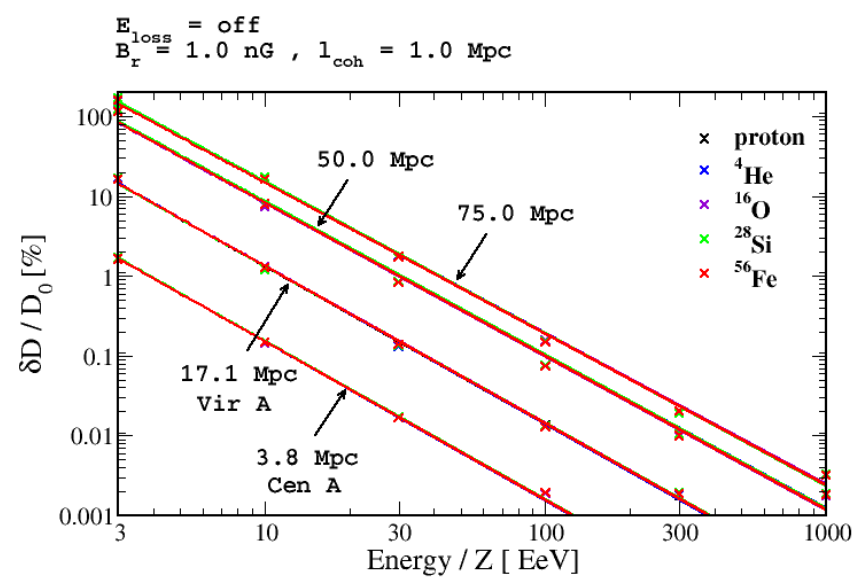

Figure 6: $\delta D / D_{0}$ as a function of the magnetic rigidity, without energy losses, for 5 different nuclei, and some selected distances of propagation, corresponding to Centaurus A, Virgo A, and two arbitrary distances. 


\section{Conclusion}

We presented a novel numerical model for the propagation of UHECRs in the EGMFs medium. We assumed a cellular structure of coherent magnetic fields: they are considered to have random orientations of their vector components within the cells with smooth transitions between them. EGCRProp presented high accuracy on calculating the particle tracks, on the order of $\lesssim 10^{-33}$ of relative error in energy, and $\sim 10^{-5} \%$ in Larmor radius for circular orbits, without energy losses. Additionally, to investigate the deviations from rectilinear propagations, we plotted the percentual distance amplification as a function of the distances travelled and of magnetic rigidities.

\section{Acknowledgments}

The authors are thankful to UFABC and to FAPESP (São Paulo State Research Foundation) for the financial and computational supports that made possible the execution of this work.

\section{References}

[1] I. Valiño for the Pierre Auger Collaboration, Proc. of $34^{\text {th }}$ ICRC, The Hague; PoS (ICRC2015) 271; arXiv:1509.03732v1 [astro-ph.HE] (2015).

[2] R. Beck and R. Wielebinski, Magnetic Fields in Galaxies, in Planets, Stars and Stellar Systems, Vol. 5, Chapter 13, Springer, Berlin (2013), arXiv:1302.5663v2 [astro-ph.GA] (2013).

[3] E.M. de Gouveia Dal Pino, Proc. of the Sobral Conf. on "The Sun, The Stars, The Universe and General Relativity", AIP (2009); arXiv:1003.3884v1 [astro-ph.CO] (2010).

[4] P.P. Kronberg, Rep. Prog. Phys. , 57, 325 (1994).

[5] D. Grasso and H. Rubinstein, Phys. Rep. , 348, 163 (2001).

[6] A. De Angelis, M. Persic \& M. Roncadelli, Mod. Phys. Lett. A23, 315 (2008)

[7] http://pesquisa.ufabc.edu.br/egcrprop/

[8] J. D. Jackson, Classical Electrodynamics (3rd ed.). New York: Wiley (1998).

[9] http://www.MPFR.org/.

[10] https://gmplib.org/.

[11] http://www.holoborodko.com/pavel/mpfr.

[12] A. Achterberg et al., arXiv:9907060v1 [astro-ph] (1999).

[13] D. Allard, Astrop. Phys., 39-40 (2012) 33, arXiv:1111.3290v1 [astro-ph.HE] (2011). 